\title{
Insights into the world of ssDNA viruses
}

\author{
V. G. Malathi ${ }^{1} \cdot$ Indranil Dasgupta $^{2}$ (1)
}

Received: 23 March 2019/Accepted: 27 March 2019/Published online: 19 April 2019

(C) Indian Virological Society 2019

In recent years, there has been dramatic increase in the discovery of single stranded (ss)DNA viruses having circular or linear genome. Metagenomic approaches and high throughput sequencing have revealed the existence of these viruses in diverse habitats such as extreme hot spirings, marine sediments, guts of invertebrates and human, faecal matter of birds and many mammals. These are the only group of viruses that can infect all three domains of life; bacteria, archaea and eukaryotes. Though ssDNA viruses infecting human beings and domesticated animals are not known to cause serious diseases, those that infect plants and shrimps are major pathogens affecting agricultural and aquaculture productivities and hence, are economically important.

Viruses having ssDNA are the smallest known, whose morphology and particle size vary. The eukaryotic viruses are icosahedral and non-enveloped; prokaryotic viruses may be filamentous, coil shaped or pleomorphic. The eukarotic viruses contain genome as small as $1-2 \mathrm{~kb}$, up to $10 \mathrm{~kb}$; whereas bacterial and archae have genomes ranging from 10 to $25 \mathrm{~kb}$. Except the members belonging to the family Parvoviridae and Bidnaviridae, all members have cicular genomes. The genome organization is simple in the

Indranil Dasgupta

indrani158@yahoo.co.in

1 Department of Plant Pathology, Tamil Nadu Agricultural University, Coimbatore, India

2 Department of Plant Molecular Biology, University of Delhi South Campus, New Delhi, India case of eukaryotic viruses with two major conserved genes, encoding coat protein and replication associated protein gene (Rep) present in either one strand or in both strands. The prokaryotic viruses have more extensive proteome and elaborate structural features. The viral ssDNA is replicated through rolling circle method (RC) in all families except the family Bidnaviridae. The Rep protein has conserved motifs of superfamily III helicase, present in all species with minor variations. PCR-based analysis of such conserved sequences enabled identifying and assembling the virus group, circular replication associated protein encoding single stranded viruses (CRESS). The simple genome of ssDNA viruses exhibit high recombination and substitution rates, resulting in the emergence of newer strains. Besides, there is also evidence of genome integration and horizontal gene transfer in eukaryotic viruses, making the study of these viruses very rewarding.

Currently, thirteen families of ssDNA viruses have been established (ICTV, http://ictv.global/report), of which eleven contain circular genome and two, Parvoviridae and Bidnaviridae have a linear single stranded DNA genome. Seven of these eleven families infecting eukaryotes are Anelloviridae, Bacilladnaviridae, Circoviridae, Geminiviridae, Genomoviridae, Nanoviridae and Smacoviridae; those infecting bacteria belong to families Microviridae, Inoviridae and those infecting archaea are grouped under two families; Pleolipoviridae and Spiraviridae. Besides viruses, the satellite DNA molecules associated with nanoviruses and geminiviruses have been reclassified into two families, Alphasatellitidae and Tolecusatellitidae.

In this special issue we have assembled information on ssDNA viruses in the form of reviews and research articles. Under the review articles, Rishishwar and Dasgupta review the accumulated information on RNA silencing suppressor proteins of begomoviruses, emerging as determinants of 
viral virulence; Ruhel and Chakraborty discuss the multifuntional Rep proteins, its interaction with host factors and its role as RNA silencing suppressor; Kushawaha and Dasgupta compare different methods of delivery of cloned DNA viral and satellite components to assess infectivity, an essential step to study viral gene functions; Malathi and Renukadevi provide an overview of discovery, taxonomy and evolutionary trends obeserved in CRESS viruses; Venkataraman and Selvarajan review the structural organization of Rep proteins of nanoviruses; Das et al. summarize the different disease manifestations of Parvovirus B-19, the only human pathogenic member of the family Parvoviridae and discuss various vaccination strategies; Mondal et al. review the molecular evolution of begomoviruses and finally, Dhar discusses the ssDNA viruses infecting shrimp and how it has affected the industry. In the research articles, Idris et al. report the emergence of merrimia mosaic virus in tomato in Puerto Rico; Hameed et al. present information on the spread of chickpea chlorotic dwarf virus in Pakistan; Abbas et al. reveal a new RNA silencing suppressor function of the replication-associated protein of an alphasatellite and finally, Marwal et al. report on the occurrence of Gravevine red blotch virus in India for the first time.

It is hoped that the reviews and research articles published in this issue serve as a good resource material on ssDNA viruses. The Editors of this special issue thank all contributors and reviewers for valuable support.

\section{Guest Editors}

Dr. V.G. Malathi is currently working as Consultant at Department of Plant Pathology, Tamil Nadu Agricultural University, Coimbatore, India. She recieved her M.Sc. and Ph.D. from Centre for Advanced studied in Botany, University of Madras, Chennai, India. In the past 25 years, she worked on characterization of begomoviruses infecting grain legumes, cotton and vegetables. Her work involved the development of infectious

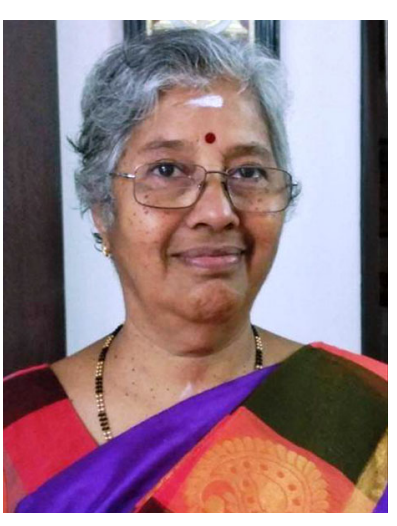

constructs, transgenic resistance and dignosis of begomo and mastreviruses. She has published about eighty research pepers in peer reviewed journals. She had been the Member of Study group on geminiviruses (2013-2016) in International Committee on taxonomy of Viruses. She is recipient of several awards such as Best woman Scientist AwardICAR for year 2010, Khwarizmi International award given by Republic of Iran (2007) and Best Teacher award given by IARI, ICAR for the year 2007 as recognition of her research and teaching achievements.

Prof. Indranil Dasgupta is a Professor at the Department of Plant Molecular Biology, University of Delhi South Campus, New Delhi, India. $\mathrm{He}$ received his M.Sc. and Ph.D. degrees from Jawaharlal Nehru University, New Delhi and post-doctoral training at John Innes Centre, Norwich, UK. His main contributions include transgenic resistance against rice tungro viruses and against begomoviruses infecting tomato, development of a virus-induced gene silencing system for rice and functional dissection of the promoter of rice tungro bacilliform virus. He has contributed to the diagnosis and molecular characterization of begomoviruses infecting cassava and okra and badnaviruses infecting citrus. He has more than sixty publications in peer-reviewed journals and has one national and one international patent related to viruses. $\mathrm{He}$ is a Fellow of all three Science Academies in India and is a recepient of J. C. Bose Fellowship of the Department of Science and Technology, Government of India. 